\title{
The impact of sleep deprivation on hippocampal-mediated learning and memory in rats
}

\author{
Saygin $\mathrm{M}^{1,2}$, Ozguner $\mathrm{MF}^{1,2}$, Onder $\mathrm{O}^{2,3}$, Doguc $\mathrm{DK}^{4}$, Ilhan $\mathrm{I}^{4}$, Peker $\mathrm{Y}^{5,6}$ \\ Department of Physiology, Faculty of Medicine, Suleyman Demirel University, Isparta, Turkey. \\ fizyolog@gmail.com
}

\begin{abstract}
BACKGROUND: To examine the impact of different types of sleep deprivation on hippocampal-mediated learning and memory in rats.

METHODS: Forty-eight Sprague-Dawley male rats were randomly assigned to 1 of 4 equal-size groups: (1) 12 hours of sleep per day (control). (2) total sleep deprivation (TSD), (3) rapid eye movement (REM) deprivation $(\mathrm{RD})$, and (4) sleep restricted to 4 hours per day (SR). All rats were subjected to swimming training in the Morris water maze (MWM). At the end of the experiments, the rats were decapitated, and hippocampus tissue was analyzed for several neurotransmitters and receptors.

RESULTS: The time spent at the target quadrant increased from 20.2 to 30.0 seconds in the control group on the third day of the experiment, whereas corresponding values increased from 20.2 to 21.8 seconds in the TSD group, 22.1 to 25.4 seconds in the RD group, and 21.2 to $32.0 \mathrm{sec}$ in the SR group $(p=0.026)$. On the seventh day of the experiment, the values decreased to 25.0 seconds in controls, 22.5 in the RD group, and 23.6 in the SR group $(p=0.045)$. The TSD group demonstrated significant decreases in glutamate and serotonin levels compared with the control group. There was a significant increase in $5-\mathrm{HT} 2$ a receptor expression in all intervention groups compared with the controls.

CONCLUSIONS: Our results of glutamate levels and 5-HT2a receptor expression in the hippocampus seem to be primarily involved in sleep and memory regulation (Tab. 2, Fig. 4, Ref. 59). Text in PDF www.elis.sk.

KEY WORDS: sleep disorders, learning and memory, 5-HT2A, NMDA, $\alpha 7$, glutamate, serotonin, acetylcholine.
\end{abstract}

\section{Introduction}

As scientists have sought to determine the function of sleep, research results have increasingly pointed to the important role that sleep plays in synaptic plasticity, reorganization of the brain, and memory processing (1). Much of this work has taken place in studies that employed sleep deprivation, the results of which have shown that learning that takes place before or after sleep deprivation results in memory deficits in both humans (2) and animals (3, 4). In rodents, the relationship between sleep and memory con-

${ }^{1}$ Department of Physiology, Faculty of Medicine, Suleyman Demirel University, Isparta, Turkey, ${ }^{2}$ Center for Sleep Sciences and Medicine Suleyman Demirel University, Isparta, Turkey, ${ }^{3}$ Department of Pulmonary Diseases, Faculty of Medicine, Suleyman Demirel University, Isparta, Turkey, ${ }^{4}$ Department of Medical Biochemistry, Faculty of Medicine, Suleyman Demirel University, Isparta, Turkey, ${ }^{5}$ Department of Molecular and Clinical Medicine, Institute of Medicine, Sahlgrenska Academy, University of Gothenburg, Gothenburg, Sweden, and ${ }^{~}$ Department of Pulmonary Medicine, Marmara University, Istanbul, Turkey

Address for correspondence: M. Saygin, Dr, PhD, Department of Physiology, School of Medicine, Suleyman Demirel University, Morfoloji Binasi, Cunur, TR-32260 Isparta, Turkey.

Phone: +90.246.2113604, Fax: +90.246.2371165

Acknowledgement: This investigation was supported by the Scientific Research Fund of Suleyman Demirel University, Project number of 3171D2-12. solidation has primarily been studied through the testing of spatial memory, which is dependent on the hippocampus (5).

A number of studies that have incorporated sleep deprivation in animals have explored the impact of potential cellular and molecular changes on learning, such as neurotransmitter levels (6), impairment of formation of long-term potentiation (cellular correlate of learning and memory;(7), and effects on levels of extracellular signal-regulated kinases (8). However, the molecular mechanism of changes in neuronal properties induced by sleep deprivation remains poorly understood.

A number of methods can be used to assess the role of sleep in learning and memory processes in animals. One is to record sleep patterns after acquisition of a learning task. Previous studies have shown that rapid eye movement (REM) sleep increases following learning sessions $(9,10)$. The synaptic activities that occur during learning initiate complex molecular cascades through the activation of different related receptors, intracellular second messengers, and transcription factors. These receptors, messengers, and transcription factors play essential roles in regulating memory-related gene expression and protein synthesis that are necessary for the acquisition of long-term memory. Sleep deprivation has a strong impact on memory: sleep deprivation that occurs before learning results in reduced learning ability and impaired memory, whereas sleep deprivation that occurs after learning impairs memory formation (11). The question is then, what happens to hippocampal cell pro- 
liferation, synaptic plasticity, and other parts of this process under conditions of chronic sleep deprivation and restriction or when an animal or human is exposed to repeated partial sleep deprivation, as more commonly occurs in modern society (12).

The activity of the neural networks within the hippocampus can be modulated by the induction of long-term potentiating, (13) as evidenced by Kim et al (2005) (14) who showed that sleep deprivation impairs long-term potentiating area CA1 of the hippocampus. The released glutamate binds to the glutamatergic $\mathrm{N}$-methyl-D-aspartate (NMDA) receptors on the postsynaptic membrane, causing a large influx of calcium ions. The increased intracellular level of calcium ions activates various kinases, including calcium-calmodulin dependent kinase II (CaMKII), which is generally known to be the regulator of short-term memory and long-term potentiation (15).

The cholinergic system and others, such as the glutamatergic, dopaminergic, and serotonergic systems, are known to be involved in learning and memory. Modulation of 1 or more of these neurochemical systems may affect different stages of learning and memory. NMDA receptors, which are 1 of the subtypes of the glutamate receptor, are known to play a key role in neural physiology, synaptic plasticity, and behavioural learning and memory (16).

The aim of the present study was to determine the effects of total sleep deprivation (TSD), REM deprivation (RD), and sleep restriction (SR) on rats' ability to function in the Morris water maze (MWM) and on nACh- $\alpha$ 7, NR2A, NR2B and 5-HT2A hippocampal receptors and glutamate, acetylcholine, and serotonin levels, which are known to be involved in learning and memory.

\section{Materials and methods}

\section{Animals}

Forty-eight Sprague-Dawley male rats, weighing 320-360 grams, were obtained from the Animal Investigation Laboratory of Suleyman Demirel University. The rats were housed individually in solid-floored cages with wood chips in a temperature-controlled $\left(23 \pm 1{ }^{\circ} \mathrm{C}\right)$ room for 1 week. Throughout the training and at the conclusion of each experimental procedure, the rats were given ad libitum access to water and food, with food quantity sufficient to maintain the rats' body weights at $80 \%$ of their free-feeding levels. On the days on which experiments took place, the food and water were provided after the testing. For 1 week prior to experimental conditions, the rats were adapted to the laboratory under 12/12-hour reverse light/dark conditions (lights off at 08:00). The animals were handled in accordance with the guidelines for animal care and experimentation of the pertinent European Communities Council Directive (86/609/EEC), and all the procedures were approved by the Ethics Committee of Suleyman Demirel University (Number: 22.11.2011/01).-

\section{Experimental groups}

After the learning period, the rats were randomly assigned to 1 of 4 equal-size groups: (1) TSD, (2) RSD, (3) sleep restricted to 4 hours per day (SR), or (4) 12 hours of sleep per day (control). From Day 6 through Day 8, rats in the TSD Group were completely deprived of sleep. From Day 6 through Day 12, the rats in the other experimental groups were exposed to RD and SR, respectively.

\section{General design}

All of the groups were trained and tested in the Morris water maze (MWM) on spatial learning and memory (17). Behavioural testing was performed during the dark phase, which is the animals' most active period (18). All rats were subjected to swimming training in the MWM float assembly (learning period) 4 times per day for 5 days. Twenty-four hours after the last training test, we implemented the TSD protocols and began conducting probe trials, hidden-platform tests, and visible-platform tests in the MWM each day for each group. Beginning on the sixth day, we implemented the TSD protocol for 3 days and the RD and SR protocols for 7 days in the respective groups. We removed the platform from the assembly for 60 -second periods (probe test): for the TSD group, we conducted the visible platform test on the day before the experiment and on Day 3 of the experiment, whereas, for the RD and SR groups, we conducted the visible platform test on the day before the experiment and on Day 7 of the experiment. We conducted a probe trial test on each day of the experiment. During this test, the platform was removed, and the rats were released from the other 3 quadrants that had not previously contained the hidden platform; the rats were allowed to swim for 60 seconds. The time spent in the target quadrant in which the platform had been during the acquisition period was recorded, and these data were used as measures of spatial memory. Thereafter, the visible platform procedure a cued version of the MWM escape task was executed every day for each group except on the final day of the experiment. We included this procedure to study whether the observed effects of the drug were direct or indirect (such as impaired vision or motivation or an increase in anxiety). Each rat was released from the fourth quadrant in each trial; the visible platform was placed in different quadrants (zones 1-3) for each trial, and the escape latency to the visible platform was recorded.

Following the last probe trial, we anesthetized all of the animals by intraperitoneally injecting $2 \%$ xylazine $(10 \mathrm{mg} / \mathrm{kg})$ and then $10 \%$ ketamine $(80 \mathrm{mg} / \mathrm{kg})$. Rats were sacrificed, and hippocampi were rapidly removed for analysis by Western blot testing.

\section{Sleep deprivation}

\section{Control Group}

Within the 4 cages of the control group, we placed three $13-\mathrm{cm}$ scale platforms. During the experimental period, the animals were provided free access to food and water. During the experiment, the control group underwent daily probe tests, and at the completion of the experiment, we performed the platform test.

\section{TSD Group}

The rats in the TSD group spent the entire 3 days of the experiment on a $3.3-\mathrm{cm}$ platform (19). They were removed from the platform only during the test periods. Memory testing was performed each day with the probe test; the platform test was conducted at the conclusion of the 3 days. 
408-416

\section{RD Group}

RD was produced with the well-established "flowerpot" technique, which consisted of placing the rats onto an inverted flowerpot (6.5-cm diameter) placed inside a large pail, which was filled with water up to $1 \mathrm{~cm}$ below the top of the flowerpot $(20,21)$. This technique has been shown to selectively deprive rats of REM but not non-rapid eye movement (NREM) sleep (22). When rats enter REM sleep, their muscle tone diminishes and causes them to touch the water, which arouses them. Because the animals can move from platform to platform within the multiplatform chamber, this device has been reported to produce less immobilization stress, as compared with the widely used single small-platform technique (23). Rats are awakened at this point, thereby terminating the REM episode, although they typically do not fall off the platform.

\section{SR Group}

Animals within the SR group were kept in normal cages and allowed ad libitum access to food and water. A typical light-dark cycle of 12 hours was applied. The rats slept less than $75 \%$ of the normal amount of 12 hours of physiological sleep (24) these experimental rats were allowed to sleep for 4 hours daily. Given that rats have an average sleep onset latency of 20.3 minutes, (25) we applied a stimulus every 15 minutes, for 5 minutes, from 00:00 to 08:00 that kept the rats awake. We continued this SR protocol for 7 days, and at the end of each day, the animals completed the probe test and post-test platform test.

\section{The MWM}

Performance in the MWM was tested in a dimly lit, soundproof room with various visual cues. The rats' task in the MWM was to find a submerged hidden platform in a pool of water. A probe trial test in the MWM was conducted 24 hours after the last training test. This was followed by hidden- and visible-platform tests, as well as a new probe trial on each day of the intervention period. The main outcomes were the time (in seconds), spent in finding the hidden platform (learning) and at the target quadrant (memory).

The MWM consisted of a circular pool, $150 \mathrm{~cm}$ in diameter and $80 \mathrm{~cm}$ in height, with the interior painted white. The water was maintained at a temperature of $22 \pm 1{ }^{\circ} \mathrm{C}$ and was made opaque by adding nontoxic dark yellow paint. The pool was surrounded by 4 halogen lights, which were directed at the walls that surrounded the room. Each trial was tracked using an overhead camera (Sony SSC-DC398P. Sony, Tokyo, Japan) interfaced with a computer that recorded the time and path traveled (Smart Version 2.0, Panlab S.L., Barcelona, Spain). The maze was divided into 4 equal-size virtual quadrants, designated as zones 1 to 4 . The platform was maintained in a fixed location for all runs, and the target quadrant was the fourth quadrant. Throughout the experiment, animals were handled before the first trial each day and then released while facing the wall of the pool from each of the 4 quadrants. Daily training consisted of 4 trials, with an intertrial interval of $\sim 20$ seconds, in which the rat was placed in the water from 4 random starting positions $(1,2,3$, or 4 ), and the latency to escape to the hidden platform was recorded. Starting locations were equally spaced around the perimeter of the pool. This MWM test was conducted for 5 consecutive days. Us- ing the protocol of Morris, (18) acquisition of place learning using spatial cues and navigational strategy were completed on days 1 through 4, at which time we conducted the test of memory, the probe trial. From Day 1 to Day 5, the animals were allowed to swim in the pool for a maximum of 60 seconds. The trial ended when the rat climbed onto the platform. On the first day, if the rat could not find the hidden platform within 60 seconds, its performance was recorded as 70 seconds, and it was placed on the platform for 30 seconds; on the following days, the resting time on the platform was decreased to 15 seconds. Rats that found the hidden platform were left on the platform for 30 seconds and then returned to their home cages by the person conducting the experiment. After the trials had been completed, the rats were dried with a towel and warmed under a 40W soft white bulb (Osram; Osram AG, Munich, Germany) before being returned to their home cages. At the end of the 5 days, each rat had undergone a total of 20 training trials. Path lengths and the time spent in locating the hidden platform were recorded for each rat. These data were used to calculate swim speeds.

\section{Tissue analysis}

At the end of the experiments, the rats were euthanized and decapitated; their brains were rapidly removed. The hippocampus was dissected on an ice packet that had been wetted with cold phosphate buffer $(50 \mathrm{mmol} / \mathrm{L})$ for further analysis of several neurotransmitters and receptors. The tissue was placed in Eppendorf tubes (Eppendorf AG; Hamburg, Germany) filled with phosphate buffer $(50 \mathrm{mmol} / \mathrm{L})$ and frozen at $-80{ }^{\circ} \mathrm{C}$ until assayed.

\section{Western blotting test}

The gel electrophoresis step is included in Western blot analysis to resolve the issue of the cross-reactivity of antibodies. AntiNR2A, anti-NR2B, anti 5-HT2A, and anti-nAch $\alpha 7$ were purchased from Millipore (Billerica, MA). Mouse monoclonal antibody to $\beta$-actin was purchased from Abcam Company (Cambridge, MA), and prestained molecular-weight marker was purchased from Sigma-Aldrich, Co. (Steinheim, Germany). All other reagents were of analytic grade or the highest grade available. Each hippocampus was weighed. The hippocampi (2 animals per preparation) were homogenized in ice-cold buffer (50 mm Tris- $\mathrm{HCl}[\mathrm{pH} 7.50 .0 .15$ $\mathrm{mol} / \mathrm{L} \mathrm{NaCl}, 1 \%$ Triton X-100, $1 \mathrm{mmol} / \mathrm{L}$ ethylenediaminetetraacetic acid [EDTA], $1 \mathrm{mmol} / \mathrm{L}$ ethylene glycol tetraacetic acid [EGTA], $25 \mathrm{mg} / \mathrm{mL}$ leupeptin, $25 \mathrm{mg} / \mathrm{mL}$ aprotinin, $1 \mathrm{mmol} / \mathrm{L}$ sodium orthovanadate, $10 \mathrm{mmol} / \mathrm{L}$ benzamidine, and $4 \mathrm{mmol} / \mathrm{L}$ p-nitrophenyl phosphate); after we obtained the supernatant fractions upon centrifuging the homogenates at $10,000 \mathrm{~g}$ for $10 \mathrm{~min}-$ utes, an aliquot was taken for protein determination. (26) Equal amounts of protein for each sample (50 $\mathrm{mg}$ of protein per lane) were separated by sodium dodecyl sulfate polyacrylamide gel electrophoresis on $7.5 \%$ minigels, blotted electrophoretically onto an immobilon membrane (Immobilon-P, Millipore), and incubated in tris-buffered saline with polysorbate 20 (Tween 20, Sigma-Aldrich, Co) (TBST; $50 \mathrm{~mm}$ tris- $\mathrm{HCl}$ [pH 7.5-8.0], $150 \mathrm{mmol} / \mathrm{L} \mathrm{NaCl}$. and $0.1 \%$ polysorbate 20 containing $3 \%$ bovine serum albumin (BSA) for 30 minutes. Blots were incubated separately overnight with anti-NR2A (1/500), anti-NR2B (1/500), anti-nACh $\alpha 7$ (1/200), 
anti-5-HT2A (1/200), and anti- $\beta$-actin (1/5000) in $1 \%$ BSA. Blots were subjected to 3 additional 10-minute washings in TBST and incubated with alkaline phosphatase-conjugated monoclonal antirabbit $\operatorname{IgG}(1 / 10000)$ in $1 \%$ BSA for 1 hour at room temperature; thereafter, 3 additional washings were performed with TBST for 10 minutes. The membrane was incubated in $20 \mathrm{~mL}$ of fresh reagent solution (5-bromo-4-chloro-3-indolyl-phosphate with nitro blue tetrazolium; BCIP/NBT) until color development. Images of immunoblots were analyzed with a computerized image-analysis system (Kodak MM 2000 Image Station; Eastman Kodak Company, Rochester; NY). SDS-PAGE and western blot analyses were carried out on 6 independent hippocampus preparations ( 2 animals per group; total of 6 preparations per group) (27). Immunoblotting for $\beta$-actin was used as an internal standard to confirm equal protein loading and sample transfer. The ratio of such subunit/ $\beta$ actin of all of the individual samples (control group and 3 other experiment groups simultaneously) was calculated for each blot. The mean of the control-group values for each antibody was set at 100 , and each value of the experimental groups was expressed as a percentage of the control-group means (28).

\section{Biochemical parameters}

All parameters were evaluated by commercial kit. Enzymelinked immunoassay, colorimetric, and ion-selective electrodes methods were used. Glutamate, acetylcholine, serotonin (Cusabio Wuhan Huamei Biotech, China) and 8-OH-2'-deoxyguanosine (Cayman, Cayman Chemical, Ann Arbor, MI) levels were measured with couples repeated by ELISA method using a commercially available kit. Thiobarbituric acid reactive substances and nitric oxide levels were measured with couples repeatedly using the colorimetric method with commercial kits (Cayman, Cayman Chemical, Ann Arbor, MI). $\mathrm{Ca}^{2+}, \mathrm{Mg}^{2+}$, and $\mathrm{Na}^{2+}$ levels were measured by the ion-selective electrodes method.

\section{Statistical analysis}

Data were analyzed with SPSS ${ }^{\circledR} 22.0$ for Windows (SPSS Inc, Chicago, IL). The Kolmogorov-Smirnov test was used to test for normal distribution of continuous variables. Data characterized by a normal distribution were expressed as mean \pm standard deviation. Parameters of nonnormal distribution were expressed as median and range. The Student t-test (normal distribution) or the Mann-Whitney test (nonnormal distribution) was used for comparing the 2 groups. Continuous variables were reported as mean \pm standard deviation, and, categorical variables, as percentages. To compare continuous variables, the Student t-test or Mann-Whitney U test was used where appropriate. Categorical variables were compared with the $\chi 2$ test. Analysis of variance (normal distribution) or the Kruskal-Wallis (nonnormal distribution) test was used for comparing more than 2 groups. First, we assessed the data for homogeneity of variance using the Levene test. MWM data were analyzed using repeated measures and 1-way analysis of variance followed by the Bonferroniposthoc test. Repeated-measure tests were used for intragroup comparisons. These data are expressed as mean \pm standard deviation, and a $p$ value of less than 0.05 was considered as statistically significant.
Because the receptor-expression data were nonhomogeneous, we used the Kruskal-Wallis test for group comparisons. When we encountered significant differences, we used the Bonferronicorrected Mann-Whitney $U$ test to determine which group was responsible for the difference. These results are expressed as mean \pm SEM, and a p value of less than 0.001 was considered to be statistically significant.

\section{Results}

\section{MWM: Learning Period}

Latency to locate the hidden platform

Day-to-day intragroup comparisons showed that MWM performances were significantly improved by Day 2 and Day 4 when compared with the performance on Day $1(p=0.01)$, indicating that the rats had learned the task. During the training period, comparison of spatial learning times between groups showed no significant differences. Latencies to find the hidden platform in all groups decreased as the experiment progressed, indicating that the rats were learning, but between-groups, the differences were not statistically significant.

\section{Memory Period}

Time spent in the target quadrant

Analysis of data from the probe trial day-to-day time spent in the target quadrant using repeated-measure tests showed no significant differences among the control, RD, and SR groups. However, time spent in the target quadrant was increased in the TSD group, as compared with the SR group on the third day $(\mathrm{p}=$ 0.001 ). Time spent in the target quadrant was significantly different when comparing the TSD and SR groups $(p=0.044)$. Within the SR group, time in the target quadrant decreased from day 3 to day $7(\mathrm{p}=0.013)$.

\section{Swim speeds}

Swim speeds were calculated by sampling path lengths $(\mathrm{cm})$ and measuring the time ( $\mathrm{sec}$ ) that each rat spent locating the hidden platform. To evaluate the swim speeds, we used data from the probe trial (Days 1-7) and visible-platform procedure (Days 0, 3, and 7). When comparing the data, distance travelled showed statistically significant differences between the TSD and SR groups on the third day $(p=0.023)$, and swim speeds increased for the TSD group. Our analysis showed a significant difference (a decrease) between Days 0 and 7 in the control $(p=0.006)$ and SR ( $p$ $=0.001)$ groups. Differences were also found between the control and RD groups $(p=0.011)$, with swim speed increased by the seventh day in the RD group and decreased in the control group. Our analysis also revealed significant differences between the RD and SR groups $(p=0.05)$, with swim speed increased on the seventh day for the RD group and decreased for SR group.

Latency to locate the visible platform

Analysis of data obtained after the learning period revealed no significant differences among groups with respect to latency to visible platform, average swimming speed, and total swimming 
408-416

Tab. 1. Receptor densities (optical density) values derived from Western blot analysis.

\begin{tabular}{lcccc}
\hline & & Groups & & \\
\cline { 2 - 4 } Receptor & TSD & RD & SR & \\
\hline NR2A & $100.81 \pm 5.94$ & $99.45 \pm 5.73$ & $102.35 \pm 4.97$ & NS \\
NR2B & $100.28 \pm 2.54$ & $100.10 \pm 3.63$ & $99.48 \pm 3.24$ & NS \\
5-HT2A & $102.02 \pm 1.96 \mathrm{~b}$ & $101.31 \pm 0.95 \mathrm{c}$ & $102.23 \pm 2.15 \mathrm{~d}$ & b: $0.029 ; \mathrm{c}: 0.014 ;$ d: 0.014 \\
nAchR $\alpha 7$ & $99.63 \pm 2.00$ & $97.92 \pm 4.46$ & $99.13 \pm 5.74$ & NS \\
\hline
\end{tabular}

Receptor densities and optical densities of all groups relative to control were calculated. b, c and d: TSD, RD and SR groups compared with control group respectively. The average optical density value of the control group is 100 . Data are presented as mean \pm standard deviation.

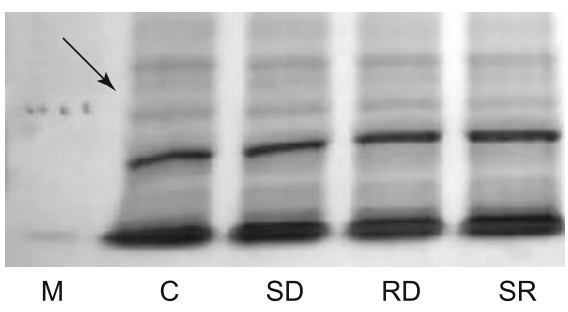

Fig. 1. Western blot analysis of the 5-HT2A receptor. $M-$ refers to marker; C - control rats; TSD - Total sleep-deprived rats; rapid eye movement (REM)-deprived rats; SR - sleep-restricted rats.

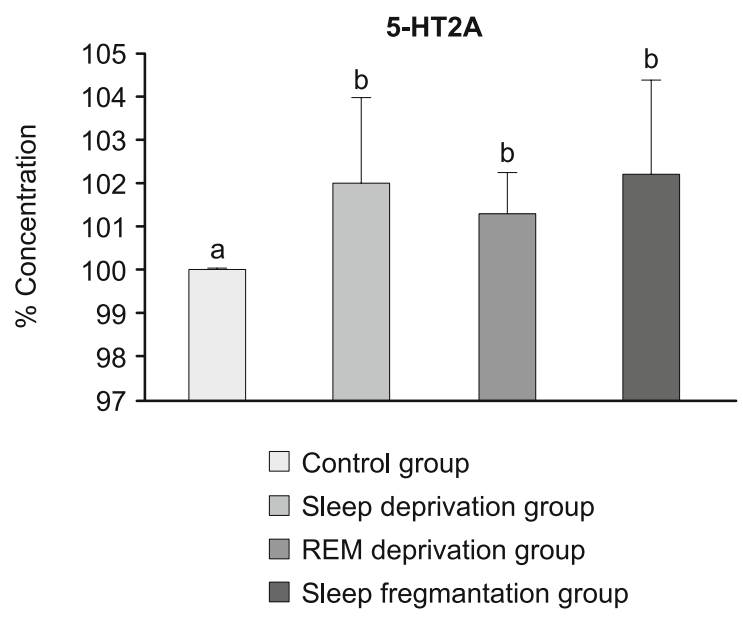

Fig. 2. Optical density results of the 5-HT2A receptor. Different characters indicate statistically significant differences compared with the mean.

time; however, within each group, average swim speed increased from day 0 to day $3(\mathrm{p}=0.05)$, and latency to visible platform ( $\mathrm{p}$ $=0.001)$, average swim speeds $(\mathrm{p}=0.000)$, and total swimming time $(p=0.032)$ differed within groups as well between days 0 and 7 .

\section{Western blot}

Using the Western blot method, we found no between-group differences in the optical densities of the glutamatergic NR2A and NR2B or $\alpha 7$ nicotinic acetylcholine (nAChR)receptors (all $p$ values $>0.05$ ); however, 5-HT2A protein expression was significantly increased in all treatment groups, as compared with in the control group (Tab. 1, Figs 1 and 2).

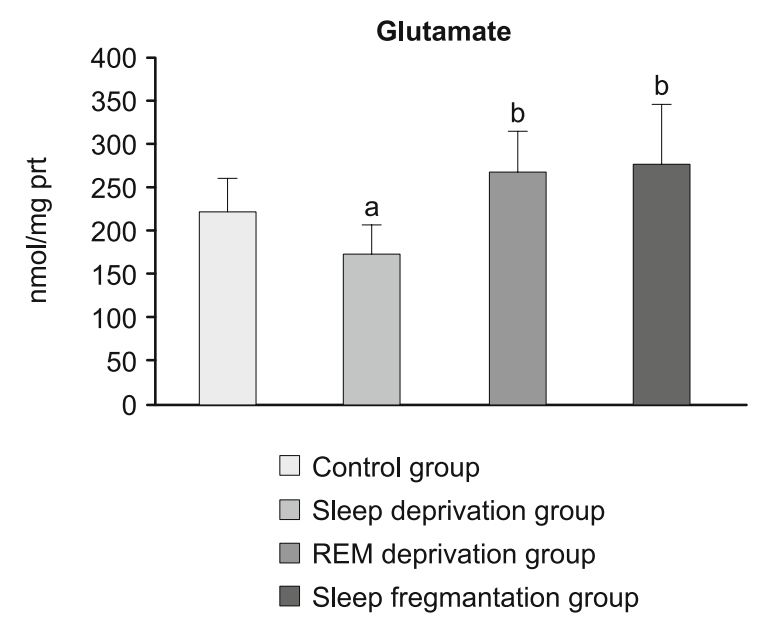

Fig. 3.Glutamate levels. Differently lettered columns indicate statistically significant differences compared with the mean.

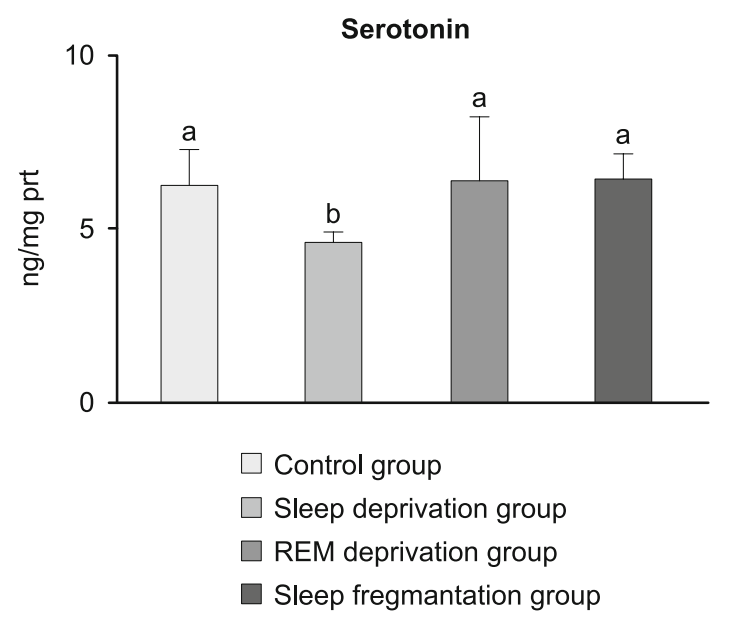

Fig. 4. Serotonin levels. Differently lettered columns indicate statistically significant differences compared with the mean.

\section{Biochemical results}

Neurotransmitters

As compared with the control group, no statistically significant differences were found in acetylcholine levels in any of the experimental groups. However, differences were found between experimental groups with regard to glutamate levels (TSD vs RD and TSD vs SR; $p=0.021$ ) (Fig. 3).Compared with the control 
Tab. 2. Levels of biochemical parameters in the hippocampus tissue ${ }^{a}$.

\begin{tabular}{|c|c|c|c|c|}
\hline Groups & Control & TSD & $\mathrm{RD}$ & SR \\
\hline Glutamate, $\mathrm{nmol} / \mathrm{mg}$ protein & $223.18 \pm 35.91$ & $176.02 \pm 31.34$ & $268.93 \pm 46.01$ & $278.51 \pm 68.01$ \\
\hline Acetylcholine, $\mathrm{ng} / \mathrm{mg}$ protein & $7.65 \pm 1.05$ & $6.48 \pm 1.42$ & $7.94 \pm 1.31$ & $6.76 \pm 1.20$ \\
\hline Serotonin, $\mathrm{ng} / \mathrm{mg}$ protein & $6.26 \pm 1.03$ & $4.64 \pm 0.30$ & $6.41 \pm 1.79$ & $6.45 \pm 0.71$ \\
\hline $\mathrm{NO} \mu \mathrm{mol} / \mathrm{mg}$ protein & $3.49 \pm 1.14$ & $3.97 \pm 1.16$ & $2.63 \pm 1.01$ & $3.25 \pm 1.32$ \\
\hline 8-OH-2' deoxyguanosine $\mathrm{pg} / \mathrm{mg}$ protein & $2002.76 \pm 413.41$ & $2108.04 \pm 387.49$ & $2025.25 \pm 473.91$ & $2245.34 \pm 195.54$ \\
\hline TBARS $\mathrm{nmol} / \mathrm{mg}$ protein & $0.44 \pm 0.19$ & $0.48 \pm 0.21$ & $0.38 \pm 0.12$ & $0.48 \pm 0.16$ \\
\hline $\mathrm{Ca}^{2+} \mu \mathrm{g} / \mathrm{mg}$ protein & $0.21 \pm 0.06$ & $0.17 \pm 0.02$ & $0.17 \pm 0.03$ & $0.17 \pm 0.06$ \\
\hline $\mathrm{Na}^{+2} \mathrm{mEq} / \mathrm{mg}$ & $0.02 \pm 0.00$ & $0.016 \pm 0.00$ & $0.016 \pm 0.00$ & $0.02 \pm 0.00$ \\
\hline
\end{tabular}

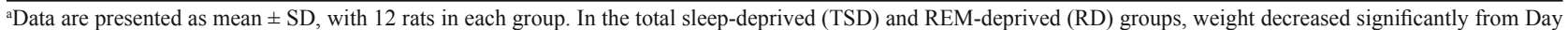
0 to Day $7(\mathrm{p}=0.001$ in both groups). SR -sleep restricted; NO - nitric oxide; TBARS - thiobarbituric acid reactive substances.

group, the TSD group had a statistically significant decrease in serotonin levels $(p=0.029)$ while the levels were increased in the $\mathrm{RD}(\mathrm{p}=0.043)$ and SR groups $(\mathrm{p}=0.021)$ (Fig. 4).

Nitric oxide, 8-OH-2'-deoxyguanosine, TBARS

$\mathrm{Ca}^{2+}$ and $\mathrm{Na}^{2+}$ levels compared were not significantly different when comparing experimental and control groups ( $p>0.05)$; however, when comparing the control and SR, we found statistically significant differences in $\mathrm{Mg}^{2+}$ levels (ie, decreased in the $\mathrm{SR}$ group; $\mathrm{p}=0.021)$. All biochemical parameters are shown in Table 2.

\section{Discussion}

Previous neuroanatomical and electrophysiological studies have clearly established the role of hippocampal formation on memory (29) with spatial memory having been shown to be strongly dependent on hippocampal activity in rats (30). The results of the current study show that sleep deprivation and sleep restriction have obvious effects on the serotonergic system of hippocampusdependent spatial learning and memory with major finding being that sleep deprivation severely impairs spatial memory related to serotonin receptor 5-HT2A.

Zhao et al (31) have shown that, when they deprived REM of rats for 3 days and then tested learning, the rats had learned the system at the beginning of the second day of the test period. Moreover, Wang et al (32) tested rats under similar circumstances and found that swimming time and distance decreased in the learning tests. These results are supportive of our findings. The measurements were affected by swimming speed of rats in water tank related to learning data. No differences were found between the swimming speeds between the groups. These measurements showed that the differences between the groups resulted from the ability of learning, but not from the swimming speeds.

Sleep deprivation did not impair nonspatial learning or memory in the visible-platform version of the MWM in our experiment. However, as compared with the rats in the control group, those in the TSD group increased their average swimming speed in the visible-platform tests. On the seventh day, the time to finding the visible platform decreased and the swimming speed increased in both the RD and SR groups, which indicates that the rats in this experiment did not lack motivation. As in the studies of Wang et al
(32) and Hajali et al (33) the rats in our TSD, RD, and SR groups had increased levels of anxiety, indicated by their faster swimming and time to find the platform.

The studies conducted to date have indicated that the neuronal circuits and activity patterns involved in the original learning process are reactivated when sleep takes place after the learning period and that this process may play important roles in memory consolidation (34).

When analysing the effect of sleep disorders on memory parameters, Guan et al (8) and McCoy et al (35) showed that, compared with the control group, those in the sleep-deprived group had decreased time in the target quadrant. Aricanli (36) found that rats that were deprived of REM sleep for 13 days spent less time in the target quadrant, compared with those in the control group, but the results were not statistically significant. Our data were similar to those of McCoy and Guan but different from Aricanli's results. Aleisa et al (37) performed 24-hour REM deprivation in 2 groups of rats with and without nicotine and showed that the nicotine group found a secret platform with significantly fewer mistakes, leading the researchers to conclude that REM deprivation impaired long-term memory and that nicotine decreased this effect. Wang et al (32) and Zhao et al's (31) study results indicated that the control group took longer to reach the target quadrant, compared with the RD group. In contrast, some studies have shown that the time to reach the target quadrant was shorter in the RD rats (33, 38 ). We suggest that the long-term memory repetition may cause these differences.

Youngblood et al $(39,40)$ found a significant difference in the swimming speed in rats after 4 days of RD compared with the control group. When Yang et al (38) deprived rats of REM sleep for 5 days, the rats swam faster, compared with rats in the control group, but the difference was not significant. The increase in swimming speed could be explained by the increased activity of the REM-deprived animals, resulting from the increased levels of sympathetic activity, irritability, and hyper excitability. These studies show that selective RD impairs the reinforcement of hippocampus-depended memory. (41).

The relationship between sleep deprivation and oxidative stress has been investigated in many studies, but the results are conflicting $(42,43)$. Serum levels of nitric oxide (NO), 8-hydroxy-2'-deoxyguanosine, and thiobarbituric acid reactive substances (TBARS) have not been found to differ significantly between animals that 


\section{8-416}

were and were not sleep deprived. Although the mechanism of NO being an important neuromodulator has not been elucidated, NO has been shown to regulate the sleep-wake cycle $(44,45)$. In our study, NO levels increased in the TSD group, results that are comparable with those of other studies. Likewise, our levels of 8-hydroxy-2'-deoxyguanosine and TBARS were comparable with those of D'Almeida et al (46). Our study did not find increased measures of oxidative stress parameters, which can be explained by the lack of exotoxicity in hippocampal cells. The changes in biochemical parameters identified by us might be related to the impaired memory functions as a consequence of sleep deprivation. In the SR group, $\mathrm{Mg}^{2+}$ levels dropped after sleep deprivation. NMDA receptor channels connected to the cation of $\mathrm{Mg}^{2+}$ binding site throughout the membrane that block by magnesium ions this connection is agonist and voltage-dependent. In this case, increased glutamate levels and NMDA-channel activation caused intracellular $\mathrm{Ca}^{2+}$ entrance and excitation. This excitation resulted in protein synthesis related to memory. Further studies are needed to understand how to modulate the ion currents of neurons to prevent the effects of sleep deprivation (47).

In our study, we found that rats in the TSD and RD groups lost weight after the experiments. Kumar and Singh (45) observed a similar weight loss sleep in their animals after 3 days of sleep deprivation, which could be explained by a stress-induced increase in energy expenditure. Yang et al (38) and Mohammed et al (48) five- and three-day studies of RD, respectively, likewise identified weight loss in rats. They attributed this weight loss to impaired physiologic mechanisms related to stress, which may induce excessive energy consumption.

The glutamate levels of rats in Mohammed et al (48) study were increased after the aforementioned 3 days of RD. When comparing control animals and those that were sleep-deprived for 24 hours, Chen et al (49) were unable to demonstrate a significant difference in NR2Aor NR2B receptors. In addition, they found a significant decrease in NMDA receptor-mediated excitatory postsynaptic potentials in hippocampal slices and hippocampus-mediated memory related to synaptic plasticity in the hippocampal NMDA receptors; their results were similar to ours.

The primary observation in our present study is that TSD, RD, and SR significantly increased 5-HT2A receptor expression in the hippocampus. 5-HT2A receptors were upregulated throughout the period of sleep deprivation. Serotonin is known to play an important role in regulating sleep and wakefulness, and the 5-HT2A receptor is involved in modulating slow-wave sleep $(50,51)$. In turn, higher serotonin levels result in downregulation of the 5-HT2A receptor, which is a $\mathrm{G}$ protein-coupled receptor. In our study, we observed an increase in 5-HT2A receptor expression in the hippocampus tissue of alternate groups, which was reflected in a change in inositol-triphosphate (IP3) and $\mathrm{Ca}^{2+}$ levels, and, in turn, resulted from the increase in $\mathrm{G}$ protein and stimulated protein kinase $\mathrm{C}$.

Basal serotonin concentrations in the rat brain are approximately $0.5 \mathrm{Nm}$ (51) and rise several-fold with sleep deprivation (52). Several studies that measured hippocampal serotonin levels by microdialysis methods showed that the maximum release in serotonin occurs during wakefulness and is decreased in NREM sleep, with further reductions occurring during REM sleep (53). Short-term sleep deprivation increases levels of 5-HT in the hippocampal cycle, with levels quickly returning to normal during sleep rebound $(53,54)$. When Senthilvelan et al deprived rats of REM sleep for 24 hours, the 5-HT cycle, measured after resting for $3 \mathrm{~h}$ and $12 \mathrm{~h}$, was increased (55).

Increases in 5-HT2A receptors reinforce learning and memory; conversely, receptor antagonists have a negative impact on learning (56). The 5-HT2A receptor antagonist volinanserin MDL 100907 (MDL) increases sleep and decreases wakefulness in rats. In addition, Morairty et al have shown that 3 different doses of MDL increase sleep in general, as well as NREM sleep and delta sleep (57). The 5-HT2A receptor indirectly controls glutamatergic signaling. Furthermore, slow wave sleep and REM sleep are generally associated with decreased glutamate levels (58), and antagonism of NMDA receptors increases delta power in NREM sleep in rats (59). With regard to the functional consequences of these regulatory processes, we found that sleep deprivation led to an increase in the 5-HT2Areceptors.

As did Lopez-Rodriguez et al (52) we found significantly decreased levels of serotonin in the hippocampus of rat brains in our TSD group, a factor that was related to increased expression of 5-HT2A receptors. However, serotonin levels were not significantly decreased in the RD group, which was explained by a decrease in serotonin secretion in NREM sleep, a reduction that continues in REM sleep (53). When Youngblood et al (39) studied the hippocampal serotonergic system in detail in rats that were sleep-deprived for 4 days and given dietary valine, they did not find the concentration of serotonin to be significantly different in the sleep-deprived versus control rats; however, an indicator of serotonin metabolism, 5-hydroxyindoleacetic acid, was significantly increased. Similarly, we found increased 5-HT2A receptors related to sleep deprivation in our rats and showed that a serotonin-related mechanism of spatial memory may be regulated by 5-HT2A receptors in the rat hippocampus.

\section{Conclusion}

We conclude that TSD as well as RD have significant adverse effects on memory, whereas short-term improvements seem to occur in rats undergoing SR. Glutamate levels and 5-HT2a receptor expression in the hippocampus seem to be primarily involved in sleep and memory regulation. These receptors mediate long-tem memory affected by TSD and RD. Circadian sleep disorders causing long-term memory impairment by LTD regulate 5-HT receptor. These phenomena might target receptor of 5-HT for long-term memory impairment related to circadian sleep disorders.

\section{References}

1. Walker MP, Stickgold R. Sleep, memory, and plasticity. Ann Rev Psychol 2006; 57: 139-166.

2. Stickgold R, James L, Hobson JA. Visual discrimination learning requires sleep after training. Nature Neurosci 2000; 3: 1237-1238. 
3. Youngblood BD, Zhou J, Smagin GN, Ryan DH, Harris RB. Sleep deprivation by the "Flower Pot" technique and spatial reference memory. Physiol Behav 1997; 61: 249-256.

4. Graves LA, Heller EA, Pack AI, Abel T. Sleep deprivation selectively impairs memory consolidation for contextual fear conditioning. Learning Memory 2003; 10: 168-176.

5. O’Keefe J, Nadel L. The Hippocampus as a Cognitive Map. Oxford: Clarendon Press, 1978; 570.

6. Hipolide DC, Moreira KM, Barlow KBL, Wilson AA, Nobrega JN, Tufik S. Distinct effects of sleep deprivation on binding to norepinephrine and serotonin transporters in rat brain. Progr Neuro-Psychopharmacol Biol Psychiat 2005; 29: 297-303.

7. Davis CJ, Harding JW, Wright JW. REM sleep deprivation-induced deficits in the latency-to-peak induction and maintenance of long-term potentiation within the CA1 region of the hippocampus. Brain Res 2003; 973: 293-297.

8. Guan Z, Peng X, Fang J. Sleep deprivation impairs spatial memory and decreases extracellular signal-regulated kinase phosphorylation in the hippocampus. Brain Res 2004; 1018: 38-47.

9. Silvestri AJ. REM sleep deprivation affects extinction of cued but not contextual fear conditioning. PhysiolBehav 2005; 84: 343-349.

10. Aleisa AM, Alzoubi KH, Alkadhi KA. Post-learning REM sleep deprivation impairs long-term memory: reversal by acute nicotine treatment. Neurosci Lett 2011; 499: 28-31.

11. Harrison Y, Horne JA. Sleep loss and temporal memory. Quart J Exp Psychol 2000; 53: 271-279.

12. Ferrara M, De Gennaro L. How much sleep do we need? Sleep Med Rev 2001; 5: 155-179.

13. Malenka RC, Bear MF. LTP and LTD: an embarrassment of riches. Neuron 2004; 44: 5-21.

14. Kim EY, Mahmoud GS, Grover LM. REM sleep deprivation inhibits LTP in vivo in area CA1 of rat hippocampus. Neurosci Lett 2005; 388: 163-167.

15. Malenka RC, Nicoll RA. Long-term potentiation - a decade of progress? Science 1999; 285 (5435): 1870-1874.

16. Woodside BL, Borroni AM, Hammonds MD, Teyler TJ. NMDA receptors and voltage-dependent calcium channels mediate different aspects of acquisition and retention of a spatial memory task. Neurobiol Learning Memory 2004; 81: 105-114.

17. Morris R. Developments of a water-maze procedure for studying spatial learning in the rat. J Neurosci Meth 1984; 11: 47-60.

18. Bettany JH, Levin ED. Ventral hippocampal $\alpha 7$ nicotinic receptor blockade and chronic nicotine effects on memory performance in the radialarm maze. Pharmacol Biochem Behav 2001; 70: 467-474.

19. Chen C, Hardy M, Zhang J, LaHoste GJ, Bazan NG. Altered NMDA receptor trafficking contributes to sleep deprivation-induced hippocampal synaptic and cognitive impairments. Biochem Biophys Res Comm 2006; 340: $435-440$.

20. Youngblood BD, Zhou J, Smagin GN, Ryan DH, Harris RB. Sleep deprivation by the "flower pot" technique and spatial reference memory. Physiol Behav 1997; 61: 249-256.

21. Kim EY, Mahmoud SG, Grover LM. REM sleep deprivation inhibits LTP in vivo in area CA1 of rat hippocampus. Neurosci Lett 2005; 388: 163-167.
22. Smith C, Gisquet-Verrier P. Paradoxical sleep deprivation and sleep recording following training in a brightness discrimination avoidance task in Sprague-Dawley rats: paradoxical effects. Neurobiol Learning Memory 1996; 66: 283-294.

23. van Luijtelaar EL, Coenen AM. Paradoxical sleep deprivation and the immobility response in the rat: effects of desipramine and phentolamine. Sleep 1985; 8: 49-55.

24. Ayas NT, White DP, Manson JE, Stampfer MJ, Speizer FE, Malhotra A et al. A prospective study of sleep duration and coronary heart disease in women. Arch Intern Med 2003; 163: 205-209.

25. Mendelson WB. Bergmann BM. Age-related changes in sleep in the rat. Sleep 1999; 22: 145-150.

26. Lowry OH, Rosebrough NJ, Farr AL, Randall RJ. Protein measurement with the folin phenol reagents. J Biol Chem 1951; 193: 265-275.

27. Delibas N, Doguc DK, Sutcu R, Eroglu E, Gokalp O. Effect of nicotine on hippocampal nicotinic acetyl choline alfa7 receptor and NMDA receptor subunits $2 \mathrm{~A}$ and $2 \mathrm{~B}$ expression in young and old rats. Internat $\mathrm{J}$ Neurosci 2005; 115: 1151-1163.

28. Liu LO, Laabich A, Hardison A, Cooper NG. Expression of ionotropic glutamate receptors in the retina of the rdta transgenic mouse. BMC Neurosci 2001; 2: 7.

29. Eichenbaum $\mathbf{H}$. The hippocampus and mechanisms of declarative memory. Behav Brain Res 1999; 103: 123-133.

30. Henninger N, Feldmann Jr RE, Futterer CD, Schrempp C, Maurer MH, Waschke KF. Spatial learning induces predominant downregulation of cytosolic proteins in the rat hippocampus. Genes Brain Behav 2007; 6: 128-140.

31. Zhao Z, Huang L, Wu H, Li Y, Zhang L, Yin Y. Neuropeptide S mitigates spatial memory impairment induced by rapid eye movement sleep deprivation in rats. NeuroReport 2010; 21: 623-628.

32. Wang GP, Huan LQ, Wu HJ, Zhang L, You ZD, Zhao ZX. Calcineurin contributes to spatial memory impairment induced by rapid eye movement sleep deprivation. NeuroReport 2009; 20: 1172-1176.

33. Hajali V, Sheibani V, Esmaeili-Mahani S, Shabani M. Female rats are more susceptible to the deleterious effects of paradoxical sleep deprivation on cognitive performance. Behav Brain Res 2012; 228: $311-318$

34. Louie K, Wilson MA. Temporally structured replay of awake hippocampal ensemble activity during rapid eye movement sleep. Neuron 2000; 29: 145-156.

35. McCoy JG, Christie MA, Kim Y, Brennan R, Poeta DL, McCarley $\mathbf{R W}$ et al. Chronic sleep restriction impairs spatial memory in rats. NeuroReport 2013; 24: 91-95.

36. Aricanli N. Selektif rem deprivasyonunun bellek fonksiyonlari üzerine etkisinde selektif serotonin reuptake inhibitörü sitalopramin etkisi. Trakya Üniveristesi Sağlik Bilimleri Enstitüsü Yüksek Lisans Tezi, Edirne, (Prof. Dr. Çetin Hakan Karadağ) 2010.

37. Aleisa AM, Alzoubi KH, Alkadhi KA. Post-learning REM sleep deprivation impairs long-term memory: reversal by acute nicotine treatment. Neurosci Letters 2011; 499: 28-31.

38. Yang RH, Hu SJ, Wang Y, Zhang WB, Luo WJ, Chen JY. Paradoxical sleep deprivation impairs spatial learning and affects membrane excitability and mitochondrial protein in the hippocampus. Brain Res 2008; 123: 224-232. 
408-416

39. Youngblood BD, Zhou J, Smagin GN, Ryan DH, Harris RB. Sleep deprivation by the "flower pot" technique and spatial reference memory. Physiol Behav 1997; 61: 249-256.

40. Youngblood BD, Smagin N, Elkins PD, Ryan DH, Harris RB. The effects of paradoxical sleep deprivation and valine on spatial learning and brain 5-HT metabolism. Physiology Behaviour 1999; 67: 643-649.

41. Rauchs G, Desgranges B, Foret J, Eustache F. The relationships between memory systems and sleep stages. J Sleep Res 2005; 14: 123-140.

42. Gopalakrishnan A, Ji LL, Cirelli C. Sleep deprivation and cellular responses to oxidative stress. Sleep 2004; 27: 27-35.

43. Khadrawy YA, Nour NA, AboulEzz HS. Effect of oxidative stress induced by paradoxical sleep deprivation on the activities of $\mathrm{Na}+, \mathrm{K}+-$ ATPase and acetylcholinesterase in the cortex and hippocampus of rat. Translat Res 2011; 157: 100-107.

44. Kumar A, Garg R. A role of nitric oxide mechanism involved in the protective, effects of venlafaxine in sleep deprivation. Behavl Brain Res 2007; 194: 169-173.

45. Kumar A, Singh A. Possible nitric oxide modulation in protective effect of (Curcuma longa, Zingiberaceae) against sleep deprivation-induced behavioral alterations and oxidative damage in mice. Phytomedicine 2008; 15: $577-586$.

46. D’Almeida V, Hipólide DC, Azzalis LA, Lobo LL, Junqueira VB, Tufik S. Absence of oxidative stress following paradoxical sleep deprivation in rats. Neurosci Lett 1997; 235: 25-28.

47. Yang RH, Wang WT, Hou XH, Hu SJ, Chen JY. Ionic mechanisms of the effects of sleep deprivation on excitability in hippocampal pyramidal neurons. Brain Res 2010; 1343: 135-142.

48. Mohammed HS, AboulEzzb HS, Khadrawyc YA, Noor NA. Neurochemical and electrophysiological changes induced by paradoxical sleep deprivation in rats. Behav Brain Res 2011; 225: 39-46.

49. Chen C, Hardy M, Zhang J, LaHoste GJ, Bazan NG. Altered NMDA receptor trafficking contributes to sleep deprivation-induced hippocampal synaptic and cognitive impairments. Biochem Biophys Res Comm 2006; 340: 435-440.
50. Popa D, Léna C, Fabre V, Prenat C, Gingrich J, Escourrou P et al. Contribution of 5-HT2 receptor subtypes to sleep-wakefulness and respiratory control, and functional adaptations in knock-outmice lacking 5-HT2A receptors. J Neurosci 2005; 25: 11231-11238.

51. Hume S, Hirani E, Opacka-Juffry J, Myers R, Townsend C, Pike V et al. Effect of 5-HT on binding of [(11)C] WAY 100635 to 5-HT(IA) receptors in rat brain, assessed using in vivo microdialysisnd PET after fenfluramine. Synapse 2001; 41: 150-159.

52. Lopez-Rodriguez F, Wilson CL, Maidment NT, Poland RE, Engel J. Total sleep deprivation increases extracellular serotonin in the rat hippocampus. Neurosci 2003; 121: 523-530.

53. Peñalva RG, Lancel M, Flachskamm C, Reul JM, Holsboer F, Linthorst AC. Effect of sleep and sleep deprivation on serotonergic neurotransmission in the hippocampus: a combined in vivo microdialysis/ EEG study in rats. Eur J Neurosci 2003; 17: 1896-1906.

54. Asikainen M, Toppila J, Alanko L, Ward DJ, Stenberg D, PorkkaHeiskanen T. Sleep deprivation increases brain serotonin turnover in the rat. NeuroReport 1997; 8 (1577): 82.

55. Senthilvelan M, Ravindran R, Samson J, Devi RS. Serotonin turnover in different duration of sleep recovery in discrete regions of young rat brain after $24 \mathrm{~h}$ REM sleep deprivation. Brain Develop 2006; 28: 526-528.

56. Harvey JA. Role of the serotonin 5-HT2A receptor in learning. Learning Memory 2003; 10: 355-362.

57. Morairty SR, Hedley L, Flores J, Martin R, Kilduff TS. Selective 5 HT2A and 5 HT6 receptor antagonists promote sleep in rats. Sleep 2008; 31: 34-44.

58. Lena I, Parrot S, Deschaux O, Muffat-Joly S, Sauvinet V, Renaud B et al. Variations in extracellular levels of dopamine, noradrenaline, glutamate, and aspartate across the sleep - wake cycle in the medial prefrontal cortex and nucleus accumbens of freely moving rats. J Neurosci Res 2005; 81: 891-899.

59. Campbell IG, Feinberg I. Comparison of MK-801 and sleep deprivation effects on NREM, REM, and waking spectra in the rat. Sleep 1999; 22: 423-432. 